\title{
The role of autophagy induced by tumor microenvironment in different cells and stages of cancer
}

\author{
Xue Yang ${ }^{1 \dagger}$, Dan-Dan Yu ${ }^{1 \dagger}$, Fei Yan ${ }^{1 \dagger}$, Ying-Ying Jing ${ }^{1}$, Zhi-Peng Han ${ }^{1}$, Kai Sun ${ }^{2}$, Lei Liang ${ }^{1}$, Jing Hou ${ }^{3}$ \\ and Li-Xin Wei ${ }^{1^{*}}$
}

\begin{abstract}
Development of a tumor is a very complex process, and invasion and metastasis of malignant tumors are hallmarks and are difficult problems to overcome. The tumor microenvironment plays an important role in controlling tumor fate and autophagy induced by the tumor microenvironment is attracting more and more attention. Autophagy can be induced by several stressors in the tumor microenvironment and autophagy modifies the tumor microenvironment, too. Autophagy has dual roles in tumor growth. In this review, we discussed the interaction between autophagy and the tumor microenvironment and the paradoxical roles of autophagy on tumor growth at different stages of tumor development.
\end{abstract}

Keywords: Autophagy, Tumor microenvironment, Tumorigenesis

\section{Introduction}

Cancer cells are surrounded by a complex milieu. This cancer cell niche is called the tumor microenvironment, and it contributes to the development and metastasis of tumors. The tumor microenvironment is a new emerging concept in tumor research and has become a research hallmark. The tumor microenvironment not only contributes to cancer cell survival by supplying nutrients but also contributes to tumor cell invasion and metastasis. Thus, detecting the composition and function of the tumor microenvironment is important for understanding the mechanisms of tumorigenesis and tumor metastasis to improve therapeutic strategies. The tumor microenvironment is a complex system and is difficult to study. It is composed of various stromal cells, including fibroblasts, vascular endothelial cells, immune cells, adipocytes, mesenchymal stems cells (MSCs), and various cytokines [1]. The physiological characteristics of the tumor microenvironment are clearly different from those

\footnotetext{
*Correspondence: weilixin_smmu@163.com

${ }^{\dagger}$ Equal contributors

'Tumor Immunology and Gene Therapy Center, Eastern Hepatobiliary Surgery Hospital, The Second Military Medical University, 225 Changhai Road, 200438 Shanghai, China

Full list of author information is available at the end of the article
}

of normal tissues and have been described as hypoxic, nutrient deprived, energy limited, acidic, and inflammatory [2-8]. These characteristics can induce autophagy by activating various pathways [5-8]; thus, autophagy can shape the tumor microenvironment. The crosstalk between autophagy and the tumor microenvironment is attracting increasing attention, as the tumor microenvironment shaped by autophagy may play a crucial role in modulating tumor development, metastasis, and therapeutic resistance.

\section{Tumor microenvironment-induced autophagy}

Autophagy is an evolutionarily conserved catabolic pathway from yeast to mammals that serves as a major lysosomal degradation pathway for recycling intracellular components. Autophagy is emerging as the key process that eliminates damaged macromolecules, including proteins, lipids, and dysfunctional organelles. Autophagy is a complex process comprising many steps (Figure 1), including initiation, elongation, and autophagosome and autolysosome formation. Macromolecules are targeted to double-membrane vesicles called autophagosomes, and autolysosomes form by fusion with lysosomes [9]. Cytoplasmic constituents are degraded and digested by lysosomal enzymes in the autolysosome for recycling 


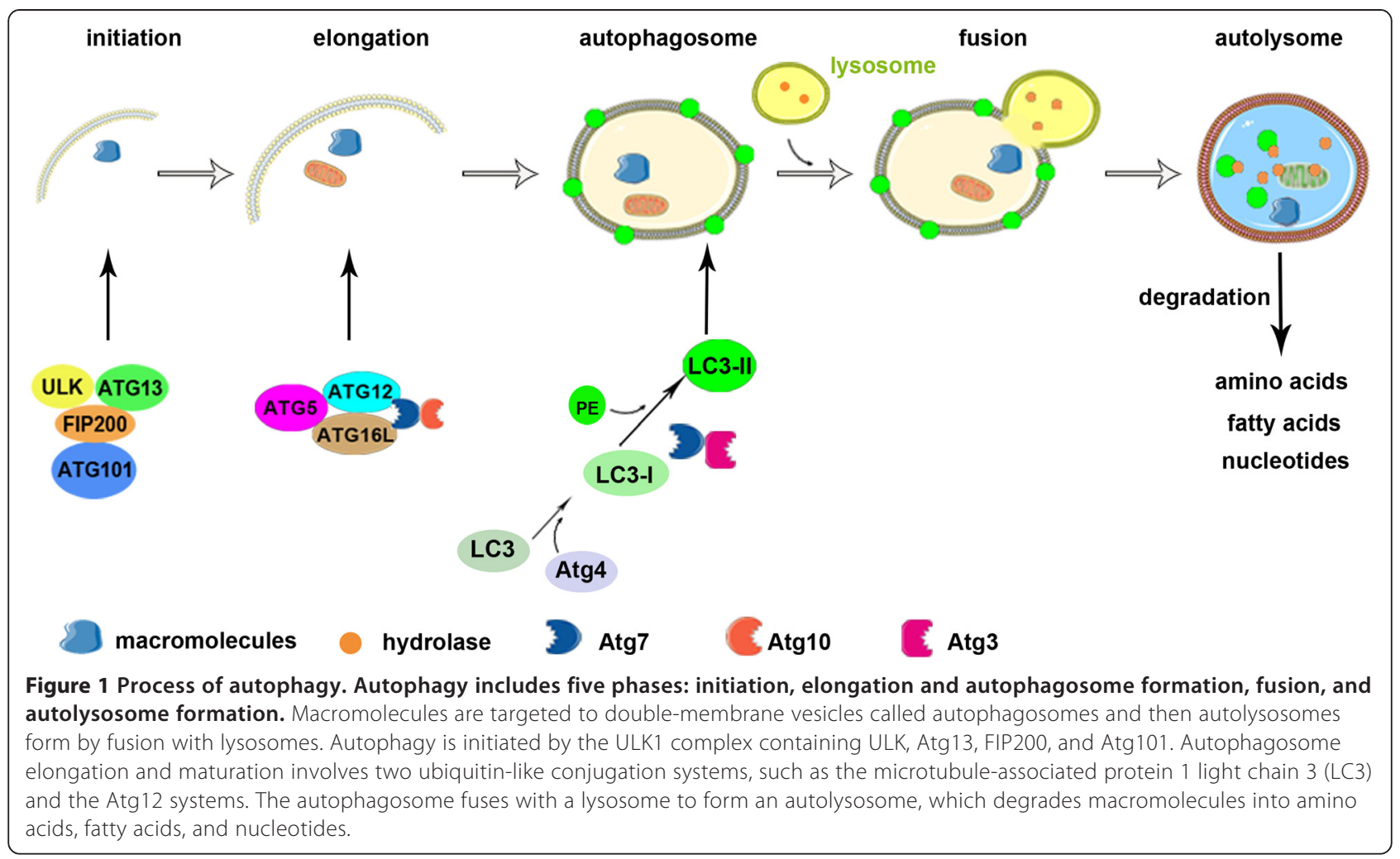

and reuse. The products of degradation, such as amino acids, fatty acids, and nucleotides, are essential for cell growth. The multi-step autophagic process is regulated by a limited number of highly conserved genes known as autophagy-related genes (ATGs) [10].

The formation of autophagosomes is initiated in mammalian cells primarily by the Unc51-like kinase 1 (ULK1) complex containing ULK, Atg13, FIP200, and Atg101 [11-13]. Activation of this complex can be inhibited by mammalian target of rapamycin (mTOR) complex 1, which is a master negative regulator of autophagy in several pathways $[14,15]$. Elongation and maturation of autophagosomes involves two ubiquitin-like conjugation systems, such as the microtubule-associated protein 1 light chain 3 (LC3) system and the Atg12 system [16]. Atg12 is conjugated to Atg5 by Atg7 (E1 enzyme) and Atg10 (E2 enzyme). The Atg12-Atg5 heterodimer interacts with Atg16L, and this complex promotes elongation of the autophagic membrane [17]. LC3I is formed immediately by Atg4B cleaving a free glycine residue after the full-length LC3 precursor is translated. After autophagy is induced, phosphatidylethanolamine $(\mathrm{PE})$ is conjugated with LC3I (called LC3II) by Atg7 (E1 enzyme) and Atg3 (E2 enzyme). PE-conjugated LC3 becomes an insoluble form (LC3-II) that is stably inserted into the autophagosomal membrane [18].

Autophagy occurs at a basal level in every cell as housekeeping and plays key roles in cell development, immunity, tissue remodeling, and orientation with the surrounding environment. In addition, in the tumor microenvironment, autophagy is also activated in response to multiple metabolic stressors (Figure 2a), such as oxygen/ nutrient deprivation, and degradation of the extracellular matrix (ECM) $[19,20]$.

Autophagy benefits cells suffering in an unfavorable microenvironment by eliminating garbage and preventing accumulation of toxins. In contrast, autophagy also supplies energy and compounds for cell survival and metabolism. Thus, autophagy serves as an inherently cytoprotective mechanism by self-eating [13].

\section{Hypoxia and anoxia}

The vasculature is insufficient to supply adequate oxygen when solid tumor diameter is $>2 \mathrm{~mm}$, resulting in local hypoxic and anoxic conditions (oxygen concentrations $<3 \%$ and $<0.1 \%$, respectively) inside the tumor [21]. Increasing evidence indicates that $50-60 \%$ of tumors grow under hypoxic conditions [22-25], and that enhanced autophagy promotes tumor cell survival [2]. Hypoxia within the immediate area of a tumor arises mainly because the balance between oxygen consumption and supply is disrupted. The supply of oxygen to normal tissues and organs meets their metabolic requirements, whereas oxygen consumption may outweigh the insufficient supply to tumors, resulting in locally low oxygen levels in tumor tissues. Hypoxia occurs in tumor 


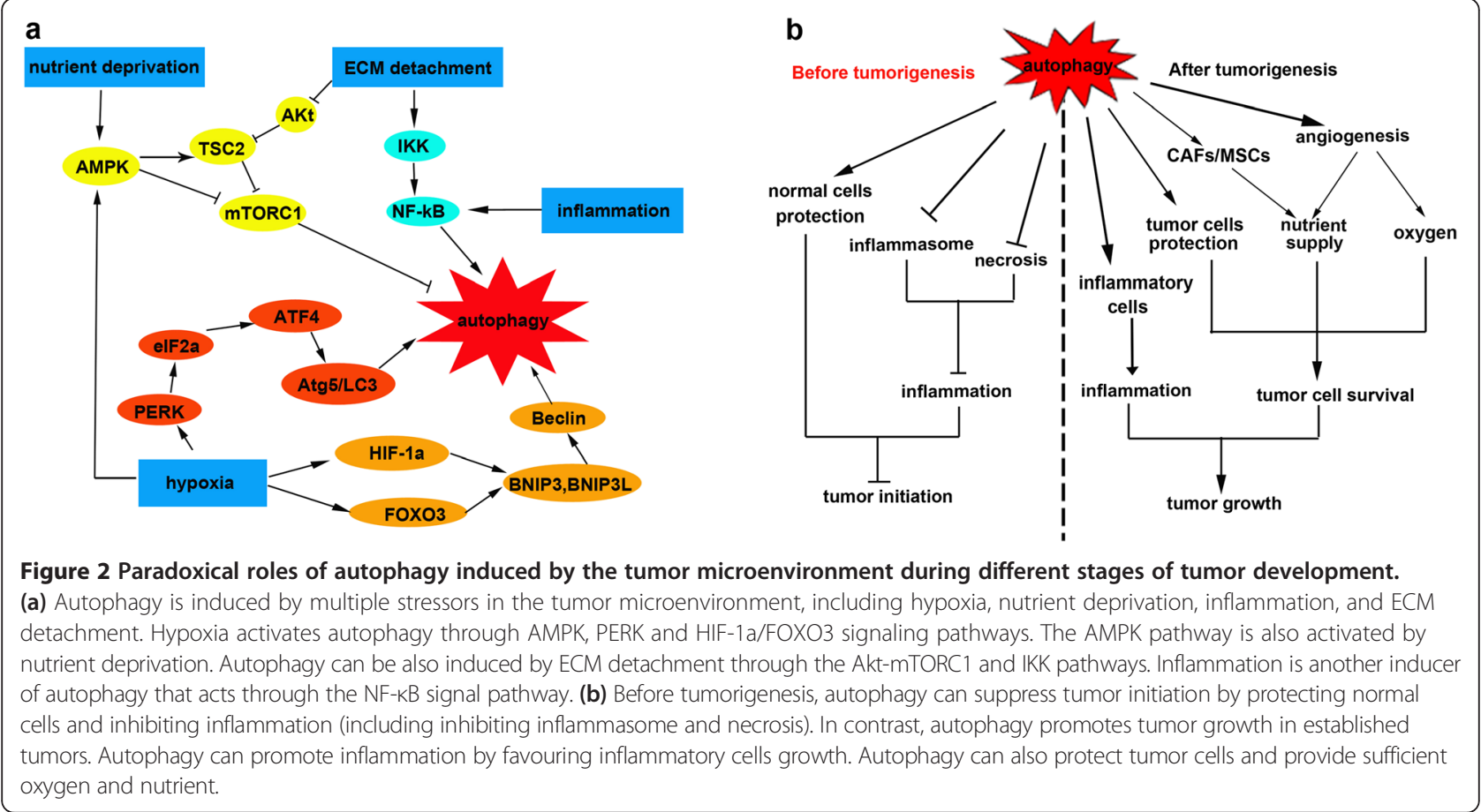

tissues for the following reasons: (1) abnormal microvessel structure and function, which is known as perfusionlimited oxygen delivery, and exists transiently; (2) limited oxygen diffusion due to increased transport distance $(>70 \mu \mathrm{m})$, which is called diffusion-limited oxygen delivery; and (3) impaired blood oxygen-carrying capacity induced by tumor-associated or therapeutic-induced anemia, which is termed anemic hypoxia [26].

Hypoxia-induced autophagy mainly depends on hypoxia-inducible factors (HIFs), whereas anoxia-induced autophagy is HIF independent [27,28]. HIFs are a family of heterodimers containing a constitutive subunit and an oxygen-regulated subunit that are only expressed when oxygen concentration declines below a 5\% threshold. HIF$1 \alpha$ activates transcription of BNIP3 and BNIP3L (BNP3like protein, also known as NIX) under moderate hypoxia, which disrupts the Beclin 1/Bcl-2 complex, releasing Beclin1 and activating autophagy [28-31], as Beclin1 is an autophagy-activator gene. Moreover, transcription of BNIP3 and BNIP3L is upregulated by the FOXO3 transcription factor. Mammucar et al. showed that FOXO3 overexpression upregulates LC3 expression and increases LC3 lipidation in skeletal muscle cells, resulting in activation of autophagy [32]. FOXO3 also plays a key role in activating hematopoietic stem cells under nutrient-deprived conditions [33]. BNIP3L, which is often present on the outer mitochondrial membrane, modulates elimination of mitochondria by autophagy (mitophagy). HIF-2 also regulates autophagy in chondrocytes and surprisingly inhibits HIF-1 $\alpha$ function [34]. Other pathways involved in hypoxia-induced autophagy include the protein DJ-1 pathway (also called CAP1/RS/PARK7), the plateletderived growth factor receptor-dependent pathway, and the unfolded protein response (always elicited by endoplasmic reticulum stress) [28,35-37]. Furthermore, inhibiting mTOR and stimulating of $5^{\prime}$ AMP-activated protein kinase (AMPK) contribute to inducing autophagy. Hypoxia-induced autophagy also requires eIF $2 \alpha$ phosphorylation mediated by PERK to reach the maximum level [38]. Hypoxia increases transcription of the essential autophagy genes LC3 and Atg5 by activating the transcription factors ATF4 and CHOP, respectively, both of which are regulated by PERK [38].

\section{Nutrient deprivation}

Proliferating cancer cells must sustain intracellular energy and nutrient levels to survive, but essential ingredients in the microenvironment at the early stage of tumorigenesis are insufficient for cancer cell survival [39]. Thus, cancer cells are nutrient deprived and to survive must cope with this stress using available metabolic pathways. Nutrient (including amino acids and glucose) depletion is the most potent physiological inducer of autophagy. Several studies have shown that autophagy plays a critical role in protecting cells against nutrient depletion [40,41]. Ammonia, generated from deamination of glutamine in mitochondria, stimulates autophagic flux in an autocrine and/or paracrine manner [42]. Amino acids, particularly branched-chain amino acids, activate mTORC1 and inhibit autophagy. Thus, the absence of amino acids induces autophagy by 
regulating mTOR activity [43]. In addition to amino acids, cells require sufficient ATP supplied by glucose, so a lack of glucose will activate autophagy to sustain energy homeostasis [44]. In addition, glucose deprivation may induce autophagy by oxidative stress [45]. Moreover, receptor for advanced glycation end product (RAGE) activates autophagy by inhibiting mTOR and blocks apoptosis in pancreatic cancer cells, which increases their survival [46]. Another report demonstrated that autophagosomes form in colorectal cancer cells under amino acid- and glucosedeprived conditions, which may contribute to survival of the cancer cells [39].

Autophagy can also be stimulated by activating the AMPK pathway to protect cancer cells against nutrient starvation. AMPK holds the core node that integrates several autophagy-inducing stimuli. The AMP: ATP ratio is a key factor for AMPK to monitor energy. Several upstream AMPK kinases, including liver kinase B1 (activated by energy depletion), calcium/calmodulin kinase kinase- $\beta$ (activated by cytosolic $\mathrm{Ca}^{2+}$ ), and transforming growth factor (TGF)- $\beta$-activated kinase-1 (involved in activating IKK) activate AMPK by phosphorylating a threonine residue on its catalytic $\alpha$-subunit [47]. The beststudied mechanisms by which AMPK induces autophagy are inhibiting mTORC1, phosphorylating tuberous sclerosis complex 2, and regulating the mTOR-associated protein, Raptor. mTOR can also be inhibited by a growth factor deficiency, such as insulin and insulin-like growth factor [21].

\section{Inflammation}

Inflammation is a cellular response that occurs at cell and tissue injury sites. Tumors were described as unhealed wounds by Dvorak over 20 years ago and were believed to produce inflammatory mediators, such as cytokines and chemokines, continuously. Tumor progression is accompanied by increased expression of inflammation-associated genes [48]. It has been widely accepted that chronic inflammation promotes cell malignancy and tumorigenesis. Bulk inflammatory cytokines, such as tumor necrosis factor (TNF)- $\alpha$, interleukin (IL)-6, TGF- $\beta$, and IL-10, contribute to the occurrence and development of cancer. In 1863, Rudolf Virchow proposed that inflammation plays a key role in tumor progression. He found that inflammatory cells infiltrate tumors [49]. His hypothesis was proved by recent studies that infection and chronic inflammationassociated disease can drive cancers [50].

The initial goal of inflammation is to eradicate foreign bodies and tumors [51]. Tumor tissues not only stimulate an adaptive immune response by recruiting $\mathrm{T}$ lymphocytes, dendritic cells (DC), and occasional B cells but also mediate the innate immune response by recruiting macrophages, polymorphonuclear leucocytes, and rare natural killer cells. However, if the inflammation cannot be controlled, surrounding tissues may be subjected to malignant transformation. Cytokines in the tumor microenvironment regulate the anti-tumor response, but cytokines induce cancer in another way during chronic inflammation [52]. Inflammation increases the risk of tumorigenesis due to the bioactive molecules secreted by cells that have infiltrated the tumor milieu, including cytokines, growth factors, and chemokines that maintain cell growth, limit apoptosis, and promote angiogenesis [53]. Furthermore, inflammation can activate autophagy [54].

Tumor cells induce oxidative stress in adjacent fibroblasts; thus, inducing nuclear factor kappa B (NF-kB) and activating HIF-1 $\alpha$, which stimulate autophagy [55-57]. Furthermore, NF- $\mathrm{kB}$ is a key regulator of the inflammatory response, suggesting a close connection between inflammation and autophagy. Ubaldo et al. showed that coculturing fibroblasts with tumor cells activates the NF- $\kappa B$ signaling pathway and induces a cytokine storm that includes IL-6, IL-8, IL-10, macrophage inflammatory protein $1-\alpha$, interferon- $\gamma$, RANTES, and granulocyte-macrophage colony-stimulating factor. Treatment with these cytokines can drive autophagy [58]. Thus, it has been speculated that inflammation plays a positive role in the occurrence of autophagy.

\section{Detachment of the ECM}

The attachment of epithelial cells to the ECM is mediated by integrin and is vital for cell growth and survival [59]. Loss of ECM attachment leads to a type of apoptosis known as anoikis [60]. Some studies have shown that a lack of appropriate ECM contact also robustly induces autophagy to promote cell survival, either during early formation of carcinoma or in the later stages of dissemination and metastasis $[61,62]$. Moreover, components of the ECM regulate autophagy and mitigate its role in cell survival, and adhesion of HeLa cells to collagen I or IV is one of the mechanisms [63]. Another study of MCF10A mammary epithelial cells (MECs) in a three-dimensional culture system showed that autophagy is rapidly induced to enhance cell survival during anoikis when cells are grown under low ECM attachment conditions [64]. Depletion of human ATGs, such as ATG5, ATG6 and ATG7, by si-RNA inhibits matrix detachment-induced autophagy [65]. Chen et al. reported that the phosphatidylinositol-3kinase (PI3K)-AKT-mTORC1 pathway is a major regulator of autophagy in detached mouse fibroblasts. Activation of the PI3K-AKT-mTORC1 pathway decreases during ECM detachment, which is correlated with the activation of autophagy. Activation of the IKK complex plays a key role promoting autophagy in MECs deprived of ECM contact [66]. Thus, both the PI3K-AKT-mTORC1 and IKK pathways are important regulators of autophagy during ECM detachment. 


\section{Autophagy shapes the tumor microenvironment}

As described before, multiple stressors drive autophagy in the tumor microenvironment, and increasing evidence shows that autophagy modifies the tumor microenvironment through different pathways. [13]. Autophagy is a key lysosomal pathway that degrades macromolecules, including intracellular organelles, denatured proteins, and nucleic acids and contributes to cell reconstruction, regeneration, and repair by recycling and reusing cellular contents. In the tumor microenvironment, autophagy may display different properties.

\section{Autophagy promotes angiogenesis}

Autophagy has important roles in tumor angiogenesis. Autophagy-associated genes in endothelial cells, such as Atg5, modulate starvation and hypoxia-evoked angiogenesis, which may occur through the high mobility group box 1 (HMGB1) pathway [67]. HMGB1 is a major chromatin-associated protein that translocates to the cytoplasm and is released from endothelial cells under stress [68]. HMGB 1 is released by damaged or dead cells and acts as an inflammatory cytokine and damagerelated protein. In addition, extracellular HMGB 1 elicits autophagy by binding to Beclin 1 [69]. HMGB 1 is an important effector of the crosstalk between endothelial cells and tumor cells and favors angiogenesis and tumor cell survival in a hypoxic microenvironment.

\section{Autophagy supplies nutrients}

Normal fibroblasts adjacent to tumor cells undergo reprogramming during tumor development by interacting with tumor cells. Fibroblasts acquire the myofibroblast phenotype and are known as cancer-associated fibroblasts (CAFs). Lisanti et al. revealed that heightened autophagy in CAFs plays a key role in energy support for neighboring epithelial tumor cells and favors their survival [70]. When CAFs overexpressing pro-autophagic molecules were co-injected with cancer cells into immunocompromised mice, they promoted tumor growth and lung metastasis. In contrast, activating autophagy in cancer cells reduces tumor growth [71]. This result suggests that CAFs supply sufficient energy for tumor cell growth by generating a fertile stroma and nutrients and that autophagy plays different roles in various compartments [72]. However, it remains unclear how the paradoxical roles of autophagy in tumors are regulated. Increasing evidence indicates that the role of autophagy in tumors is far more complex than thought previously [13].

Suppressing autophagy in apoptosis-deficient tumor cells impairs their survival under stress conditions in vivo and in vitro, indicating that autophagy sustains cell survival when nutrients are limited [2]. Cells undergoing autophagy provide nutrition by degrading intracellular proteins and organelles. Autophagy ensures metabolism of hematopoietic stem cells during trophic factor deprivation [73]. Moreover, autophagy sustains nutrient metabolism when nutrient levels are low during mouse development [74]. All of these results show that autophagy favors cell metabolism and energy balance. In contrast, defects in autophagy increase the incidence of many cancers, such as human breast, ovarian, and prostate cancers [75-77].

\section{Autophagy regulates the inflammatory response}

Growing evidence in the last 10 years suggests that inflammation plays a key role in tumor occurrence and development. Autophagy has been also proposed to be a key regulator of inflammation through various mechanisms, as an autophagy deficiency increases necrosis and inflammation in tumor cells, whereas activating autophagy has the opposite effect. White's team demonstrated in 2006 that impaired apoptosis and autophagy induces necrosis; thus, stimulating the inflammatory response and accelerating tumor growth [21]. All of these results indicate that autophagy plays a key role in cell death and inflammation induced by necrosis.

Unlike cell apoptosis, necrosis provokes an inflammatory storm. HMGB1 released from necrotic cells activates NF- $\mathrm{kB}$ after binding to the cell surface receptor RAGE $[78,79]$. Nucleic acids from necrotic cells elicit inflammation through a Toll-like receptor. Several studies have shown that autophagy blocks two forms of necrotic cell death, such as necroptosis and poly-ADP-ribose polymerase (PARP)-mediated cell death. Necroptosis is dependent on caspase and induced by a cell death ligand, such as TNF- $\alpha$ and FasL. PARP-mediated cell death is another form of programmed-necrotic cell death that can be induced by DNA damage $[80,81]$. In contrast, inhibiting the autophagy-related genes Atg5 and Beclin 1 results in increased sensitivity of cells to necrotic death [82] IL- $1 \alpha$ released from necrotic cells activates Kupffer cells, which produce cytokines, induce TNF- $\alpha$, IL-6, and hepatocyte growth factor and activate NF- $\mathrm{kB}$, leading to hepatocarcinogenesis [83]. Massey et al. showed that a deficiency in Atg16L1 is involved in Crohn's disease, suggesting a potential role of autophagy in promoting inflammation [84]. Autophagy regulates inflammatory signals directly. Inflammasomes can activate under autophagy-deficient conditions and promote maturation of inflammatory cytokines including IL-1 $\beta$ and IL-18 [85]. Further studies showed that mitochondrial reactive oxygen species (mtROS) produced by damaged mitochondria play a crucial role in this process. ROS activate the NLRP3 inflammasome, which promotes caspase 1 maturation. Activated caspase 1 cleaves pro-IL-1 $\beta$ to produce matures IL-1 $\beta$ that is subsequently secreted by cells [86]. In addition, mtROS also act as signaling molecules to trigger other inflammatory cytokines, such as TNF- $\alpha$ and IL-6 [87]. Furthermore, autophagy affects 
immune cells directly. Neutrophils, as the first immune cells migrating to a tumor inflammatory site, promote inflammation and activate macrophages and DCs [88]. The activation of autophagy in neutrophils mediates death of neutrophils, which results in decreased inflammation [89]. In contrast, antigen-presenting cells (APCs), such as macrophages and DCs, undergo autophagy to survive under stress conditions [90].

Autophagy stimulates the innate and adaptive immune responses. Autophagy is involved in activating DCs [91,92]. Autophagy in T cells has also been investigated. Autophagy is activated in both CD4+ and CD8+ T cells to promote their proliferation [93,94]. CD4+ and CD8+ cells deficient in ATG3, ATG5 and ATG7 cannot proliferate after they are activated $[95,96]$, and Atg5-/- lymphocytes fail to repopulate the periphery due to overwhelming cell death [93]. Autophagy in APCs and T cells promotes the inflammatory response.

\section{Paradoxical roles of autophagy during different stages of tumor development}

Tumor microenvironment-driven autophagy have different roles in different stages of tumor development $[2,3,97]$ (Figure 2b), the mechanism of which remains unclear. There has been work suggesting that autophagy is activated in different cells during different stages of tumor development, thus results in different effects on tumor growth.

\section{Autophagy inhibits early tumorigenesis}

Autophagy was initially considered to be a process that suppressed malignant transformation. The first direct evidence of the relationship between autophagy and cancer was established in 1999, when Levine et al. discovered that Beclin1 is a candidate tumor suppressor gene [77]. They found that Beclin1 is monoallelically deleted in a high percentage of human breast and ovarian cancers, and that Beclin1 expression is frequently low in human breast cancer, including cell lines and cancer tissues. Moreover, increased Beclin1 expression in breast cancer cell lines inhibits cell proliferation in vitro and tumor generation in nude mice [77]. Then, the importance of single copy loss of the Beclin1 gene was exhibited in Beclin1 heterozygous knockout mice, which are prone to develop spontaneous lymphomas, lung cancers, and liver cancers, as well as accelerated hepatitis B virus-induced hepatocarcinogenesis [75]. Levine et al. further found that Akt suppresses autophagy by mTOR-independent phosphorylation of Beclin1 and ultimately promotes tumorigenesis [98].

Many other autophagy machinery components besides Beclin1 play tumor-suppressive roles in tumorigenesis. Atg4C knockout mice have increased susceptibility to develop carcinogen-induced fibrosarcomas [99]. UV radiation resistance associated gene and Bif- 1 , which are components of the Beclin1/class III PI3K complex, also participate in controlling cell proliferation and suppressing tumorigenesis [100,101]. Notably, Atg5 mosaic-deleted mice develop spontaneous benign liver tumors, but no tumors are detected in other organs. Liver-specific deletion of Atg7 also leads to benign liver tumors in a mice model [102]. Atg5 -/- or Atg7-/- mice or mice hypomorphic for Atg16L1 exhibit intestinal Paneth cell abnormalities resembling Crohn's disease, which may results in intestine cancer [103,104]. Atg4, Atg 5, Atg 12 and Atg 9b have also been demonstrated to be deleted or mutated in various human cancers [7]. These reports suggest that tumor suppression may be a property of the autophagy machinery but is not associated with a signal autophagy protein. Tumors in both studies were benign hepatic adenomas but not frank cancer, suggesting that loss of autophagy may be a trigger for primary tumorigenesis, but not for malignant progression during late tumorigenesis.

\section{Autophagy promotes established tumor growth}

In addition to tumor suppression role of autophagy in the initial process of tumorigenesis, autophagy seemingly plays an opposite role as a tumor promoter in established cancers. Several studies have shown that autophagy promotes survival of tumor cells under several stressors [21]. Degenhardt et al. showed that activation of autophagy in evolving tumors promotes tumor survival [2]. Another study by Sun suggested that autophagy suppresses hepatocarcinogenesis during the dysplastic stage and promotes hepatocarcinogenesis at the tumor-forming stage [105]. Except for the difference in tumor type, this strange phenomenon may result from differences in the incipient cells involved with tumor development. Altman et al. found that a deficiency of autophagy following deletion of Atg3 aggravates BCRAbl-expressing hematopoietic precursor cell death under stress and prevents BCR-Abl-mediated leukemogenesis [106]. White's group also found that Ras expression upregulates basal autophagy, which was required for survival of immortal mouse kidney epithelial cells during starvation and during Ras-mediated tumorigenesis [107]. A study in a conditional FIP200-knockout mouse model showed that inhibiting autophagy retards MMTV-PyMT-mediated tumorigenesis of mammary epithelial cells by impairing tumor cell survival and proliferation [108].

The process of tumorigenesis involves activation of various oncogenes and inactivation of anti-oncogenes. Autophagy may mainly impact tumor cells and consequently play a role as a tumor promoter during oncogene-mediated tumor development. However, it appears that the protumorigenic role of autophagy extends beyond the hypoxia/ nutrient deprived regions of a tumor. Detachment of the ECM during early carcinoma formation or in the later stages of dissemination and metastasis robustly induces 
autophagy to promote cell survival. Autophagy also mediates therapeutic resistance in a variety of situations [97]. Tumor cells can survive after chemo- or irradiation therapy by activating autophagy. Li et al. showed that inhibiting autophagy with 3-methyladenine or by targeting Atg7 enhances the 5-fluorouracil treatment effect in human colorectal cancer cells [109]. Inhibiting autophagy also enhances the therapeutic efficacy of cisplatin and 5fluorouracil in esophageal and colon cancer cells, respectively $[109,110]$. The autophagy inhibitor chloroquine (CQ) and hydroxychloroquine (HCQ) have been used to enhance the anti-tumor effects of toxic drugs in clinical trials. Furthermore, autophagy may be a key modulator of tumor metastasis [111] by regulating the epithelialmesenchymal transition, which can be induced in the tumor microenvironment.

\section{Autophagy has different roles in different cells Normal cells}

Normal cells face the unfavourable microenvironment and are involved in metabolic stress in early stage of tumorigenesis. Metabolic stressors distinctly impact on cellular genome stability. Metabolic stressors disturb the mechanisms of DNA synthesis/repair by accumulating misfolded and aggregate-prone proteins, and reactive oxygen species (ROS)-generating organelles, especially mitochondria. In autophagy-competent cells, autophagy clears these accumulations to limit the metabolic stress. In contrast, autophagy defection sensitizes normal cells to metabolic stress, and results in DNA damage increase, gene amplification and aneuploidy increase, and ultimately promotes tumorigenesis $[3,112]$. These data showed that autophagy plays an important role in the protection of genomic stability in normal cells and inhibiting tumorigenesis. In addition, autophagy deficiency leads to differentiation disorder and abnormal proliferation of stem cells, both of which may be the early events in the process of tumorigenesis $[113,114]$.

\section{Cancer cells}

It has been known that autophagy not only benefits normal cells suffering from metabolic stress, but also protects cancer cells in the stress microenvironment during tumor development. In the rapidly growing stage of tumor development, angiogenesis can not satisfy the great demand of amino acids, oxygen and growth factors for fast-proliferating tumor cells. Autophagy can digest damaged proteins, organelles and other macromolecules and recycle cytoplasmic materials to balance the demand of nutrients and energy [115]. And a series of metabolic stressors (including starvation, hypoxia, and ROS accumulation) induce autophagy for survival in tumor cells [116]. In addition to metabolic stress-activated autophagy, autonomous autophagy also plays a crucial role in tumor development. Recently, many studies have shown that several types of tumor cells require autonomous autophagy for tumor growth in normal condition $[107,117]$.

\section{Tumor-associated mesenchymal stem cells}

In tumors, there are various cell types constituting the tumor stroma [13]. Mesenchymal stem cells is an important component of tumor stroma and have multiple roles in the tumor microenvironment [118]. The effect of MSCs on tumor cells in stressful conditions has also been described. MSCs promote survival and drug resistance in various hematological tumors [119-121]. Recent studies have established that MSCs provide sufficient stromal support for tumor cells [122]. The effect of MSCs on tumor growth in the tumor microenvironment may be related to activating autophagy. Cecilia et al. demonstrated that serum-deprived MSCs (SD-MSCs) express Beclin1, ATG10, and ATG12 and the SD-MSCs undergoing autophagy provide the needed nutrients and secrete survival and anti-apoptotic factors for selfsurvival and survival of surrounding cells [123].

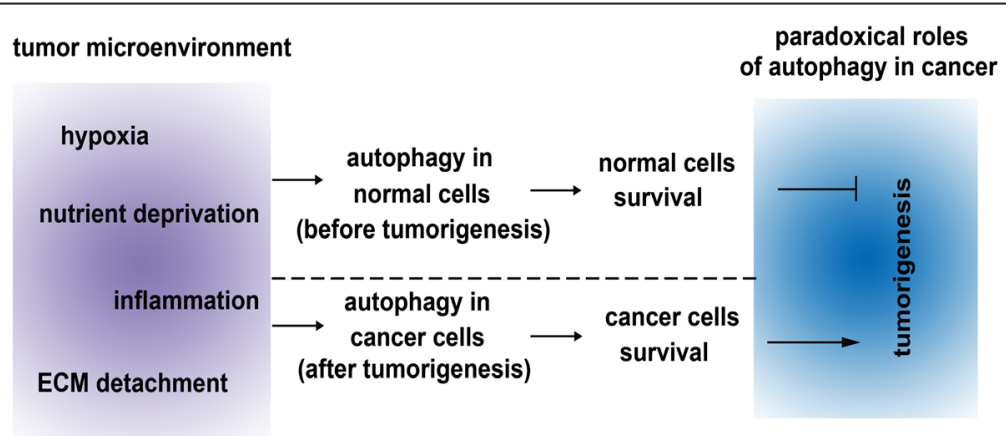

Figure 3 Concise summary of relation between autophagy and tumor growth. Autophagy can be induced by different metabolic stressors in the tumor microenvironment including hypoxia, nutrient deprivation, inflammation and ECM detachment. Autophagy can be activated in different cells at different stages of tumor growth and have paradoxical roles in tumor growth. Before tumorigenesis, autophagy promotes normal cells survival and suppresses tumorigenesis. In contrast, after tumorigenesis, autophagy promotes survival of the cancer cells and thus promotes tumor growth. 


\section{Conclusion}

Tumor microenvironment is very important to tumor growth, metastasis and therapy resistance and attracts more and more attention. Studies have shown that autophagy is an important factor in the tumor microenvironment. The mechanism of autophagy interfaced with tumor microenvironment still remains unclear and needs to be explored. But previous studies suggested that autophagy is not only a result of the tumor microenvironment, but also has effect on it. The tumor microenvironment can activate autophagy through different pathways and autophagy can modify the tumor microenvironment by promoting angiogenesis, supplying nutrients, and modulating the inflammatory response and thus help the cells in the tumor microenvironment overcome metabolic stress, maintain homeostasis, and survive in poor microenvironment.

Tumor resistance to radiotherapy and chemotherapy is also associated with autophagy. Various tumor-associated animal models have confirmed that autophagy inhibition has the effects of enhancing chemosensitivity and promoting tumor regression. Of the known autophagy inhibitors, only CQ/HCQ have been evaluated in human clinical trials [124].There have been more than 50 clinical trials working on CQ/HCQ efficacy in various human cancers, particularly in advanced or metastatic cancers (https:// clinicaltrials.gov). A combination of an autophagy inhibitor with a toxic drug possibly is an alternative treatment for advanced or metastatic cancer, and HCQ may be preferred to CQ due to its more acceptable side effects [125]. However, autophagy-targeted therapy still should be cautious because autophagy has dual roles in tumor growth progress (Figure 3). The dual roles of autophagy in tumor growth may be tissue-dependent and vary among different stages of tumor growth. Thus, autophagy inhibitor may play different roles in different stages and types of tumor growth. In addition, excess and sustained autophagy leads to tumor cell death or senescence, which results in tumor dormancy. Therefore, using autophagy as a tumor treatment target should be further investigated and studying autophagy in tumor microenvironment is very important for discovery of new therapeutic targets.

\begin{abstract}
Abbreviations
ATG: Autophagy-related genes; mTOR: Mammalian target of rapamycin complex; LC3: Light chain 3; PE: Phosphatidylethanolamine; ECM: Extracellular matrix; HIFs: Hypoxia-inducible factors; RAGE: Receptor for advanced glycation end product; AMPK: AMP-activated protein kinase; APC: Antigen-presenting cell; DC: Dendritic cells; IL: Interleukin; HMGB1: High mobility group box 1; CAFs: Cancer-associated fibroblasts; ROS: Reactive oxygen species; MSCs: Mesenchymal stem cells; ULK1: Unc51-like kinase 1; TNF-a: Tumor necrosis factor-a; NF-kB: Nuclear factor kappa B; MEC: Mammary epithelial cell; PARP: Poly-ADP-ribose polymerase; CQ: Chloroquine; HCQ: Hydroxychloroquine.
\end{abstract}

\section{Competing interests}

The authors declare that they have no competing interests.

\section{Authors' contributions}

$X Y, D D Y, F Y, Y Y J, Z P H, K S, L L, J H$ and LX W planned the manuscript outline. $X Y, D D Y, F Y$ wrote the draft manuscript, YYJ, ZPH, KS, LL, JH revised the manuscript, LXW finalized the manuscript. All authors have read and approved the final manuscript.

\section{Acknowledgements}

Key Basic Research Project of China (Grant NO. 2012CBA01303,

2011CB966200); National Natural Science Foundation of China (Grant NO. 31171321, 81372330,81372312,81201584, 81402454,81372312, 81301715); Special Funds for National key Sci-Tech Sepcial Project of China (Grant NO. 2012ZX10002-016, 2012ZX10002011-011); Shanghai Science and Technology Committee (Grant NO. 12ZR1454200, 12ZR1439800, 12431900802); Shanghai Municipal Commission of Education (Grant NO. 14ZZ086,14YZ041) and Science Fund for Creative Research Groups, NSFC, China (Grant NO. 81221061).

\section{Author details}

${ }^{1}$ Tumor Immunology and Gene Therapy Center, Eastern Hepatobiliary Surgery Hospital, The Second Military Medical University, 225 Changhai Road, 200438 Shanghai, China. ${ }^{2}$ Central laboratory, Ren Ji Hospital, School of Medicine, Shanghai JiaoTong University, Shanghai, China. ${ }^{3}$ Department of Pharmacy, Chang Hai Hospital, The Second Military Medical University, Shanghai, China.

Received: 29 December 2014 Accepted: 13 March 2015

Published online: 28 March 2015

\section{References}

1. Hall B, Dembinski J, Sasser AK, Studeny M, Andreeff M, Marini F. Mesenchymal stem cells in cancer: tumor-associated fibroblasts and cell-based delivery vehicles. Int J Hematol. 2007;86(1):8-16.

2. Degenhardt K, Mathew R, Beaudoin B, Bray K, Anderson D, Chen G, et al. Autophagy promotes tumor cell survival and restricts necrosis, inflammation, and tumorigenesis. Cancer Cell. 2006;10(1):51-64.

3. Mathew R, Kongara S, Beaudoin B, Karp CM, Bray K, Degenhardt K, et al. Autophagy suppresses tumor progression by limiting chromosomal instability. Genes Dev. 2007;21(11):1367-81.

4. Wojtkowiak JW, Rothberg JM, Kumar V, Schramm KJ, Haller E, Proemsey JB, et al. Chronic autophagy is a cellular adaptation to tumor acidic $\mathrm{pH}$ microenvironments. Cancer Res. 2012;72(16):3938-47.

5. Amaravadi RK, Lippincott-Schwartz J, Yin XM, Weiss WA, Takebe N, Timmer $W$, et al. Principles and current strategies for targeting autophagy for cancer treatment. Clin Cancer Res. 2011;17(4):654-66.

6. Chen N, Karantza V. Autophagy as a therapeutic target in cancer. Cancer Biol Ther. 2011;11(2):157-68.

7. Liu EY, Ryan KM. Autophagy and cancer-issues we need to digest. J Cell Sci. 2012;125(Pt 10):2349-58.

8. White E. Deconvoluting the context-dependent role for autophagy in cancer. Nat Rev Cancer. 2012;12(6):401-10.

9. Hoyer-Hansen $M$, Jaattela M. Autophagy: an emerging target for cancer therapy. Autophagy. 2008;4(5):574-80.

10. Apel A, Zentgraf H, Buchler MW, Herr I. Autophagy-A double-edged sword in oncology. Int J Cancer. 2009;125(5):991-5.

11. Mercer CA, Kaliappan A, Dennis PB. A novel, human Atg13 binding protein, Atg101, interacts with ULK1 and is essential for macroautophagy. Autophagy. 2009:5(5):649-62.

12. Jung CH, Jun CB, Ro SH, Kim YM, Otto NM, Cao J, et al. ULK-Atg13-FIP200 complexes mediate mTOR signaling to the autophagy machinery. Mol Biol Cell. 2009;20(7):1992-2003.

13. Maes H, Rubio N, Garg AD, Agostinis P. Autophagy: shaping the tumor microenvironment and therapeutic response. Trends Mol Med. 2013;19(7):428-46.

14. Jung $\mathrm{CH}$, Ro SH, Cao J, Otto NM, Kim DH. mTOR regulation of autophagy. FEBS Lett. 2010;584(7):1287-95.

15. Kim J, Kundu M, Viollet B, Guan KL. AMPK and mTOR regulate autophagy through direct phosphorylation of UIk1. Nat Cell Biol. 2011;13(2):132-41.

16. Geng J, Klionsky DJ. The Atg8 and Atg12 ubiquitin-like conjugation systems in macroautophagy. 'Protein modifications: beyond the usual suspects' review series. EMBO Rep. 2008;9(9):859-64. 
17. Mizushima N, Kuma A, Kobayashi Y, Yamamoto A, Matsubae M, Takao T, et al. Mouse Apg16L, a novel WD-repeat protein, targets to the autophagic isolation membrane with the Apg12-Apg5 conjugate. J Cell Sci. 2003;116(Pt 9):1679-88

18. Kabeya Y, Mizushima N, Ueno T, Yamamoto A, Kirisako T, Noda T, et al. LC3, a mammalian homologue of yeast Apg8p, is localized in autophagosome membranes after processing. EMBO J. 2000;19(21):5720-8.

19. Kondo $Y$, Kanzawa $T$, Sawaya $R$, Kondo $S$. The role of autophagy in cancer development and response to therapy. Nat Rev Cancer. 2005;5(9):726-34.

20. Yang K, Fang H, Gong J, Su L, Xu W. An overview of highly optically pure chloramphenicol bases: applications and modifications. Mini Rev Med Chem. 2009;9(11):1329-41.

21. Kroemer G, Marino G, Levine B. Autophagy and the integrated stress response. Mol Cell. 2010;40(2):280-93.

22. Vaupel $P$, Hockel M, Mayer A. Detection and characterization of tumor hypoxia using pO2 histography. Antioxid Redox Signal. 2007;9(8):1221-35.

23. Vaupel P, Mayer A. Hypoxia and anemia: effects on tumor biology and treatment resistance. Transfus Clin Biol. 2005;12(1):5-10.

24. Vaupel P, Briest $\mathrm{S}$, Hockel M. Hypoxia in breast cancer: pathogenesis, characterization and biological/therapeutic implications. Wien Med Wochenschr. 2002;152(13-14):334-42.

25. Vaupel P, Mayer A, Hockel M. Tumor hypoxia and malignant progression. Methods Enzymol. 2004;381:335-54

26. Vaupel P. Tumor microenvironmental physiology and its implications for radiation oncology. Semin Radiat Oncol. 2004;14(3):198-206.

27. Majmundar AJ, Wong WJ, Simon MC. Hypoxia-inducible factors and the response to hypoxic stress. Mol Cell. 2010;40(2):294-309.

28. Mazure NM, Pouyssegur J. Hypoxia-induced autophagy: cell death or cell survival? Curr Opin Cell Biol. 2010;22(2):177-80.

29. Mazure NM, Pouyssegur J. Atypical BH3-domains of BNIP3 and BNIP3L lead to autophagy in hypoxia. Autophagy. 2009;5(6):868-9.

30. Sandoval H, Thiagarajan P, Dasgupta SK, Schumacher A, Prchal JT, Chen M, et al. Essential role for Nix in autophagic maturation of erythroid cells. Nature. 2008:454(7201):232-5.

31. Bellot G, Garcia-Medina R, Gounon P, Chiche J, Roux D, Pouyssegur J, et al. Hypoxia-induced autophagy is mediated through hypoxia-inducible factor induction of BNIP3 and BNIP3L via their BH3 domains. Mol Cell Biol. 2009:29(10):2570-81.

32. Mammucari C, Milan G, Romanello V, Masiero E, Rudolf R, Del Piccolo P, et al. FoxO3 controls autophagy in skeletal muscle in vivo. Cell Metab. 2007;6(6):458-71

33. Warr MR, Binnewies M, Flach J, Reynaud D, Garg T, Malhotra R, et al. FOXO3A directs a protective autophagy program in haematopoietic stem cells. Nature. 2013;494(7437):323-7.

34. Srinivas V, Bohensky J, Zahm AM, Shapiro IM. Autophagy in mineralizing tissues: microenvironmental perspectives. Cell Cycle. 2009;8(3):391-3.

35. Kaushik S, Singh R, Cuervo AM. Autophagic pathways and metabolic stress, Diabetes Obes Metab. 2010;12 Suppl 2:4-14.

36. Rouschop KM, Wouters BG. Regulation of autophagy through multiple independent hypoxic signaling pathways. Curr Mol Med. 2009;9(4):417-24.

37. Pouyssegur J, Dayan F, Mazure NM. Hypoxia signalling in cancer and approaches to enforce tumour regression. Nature. 2006;441(7092):437-43.

38. Rouschop KM, van den Beucken T, Dubois L, Niessen H, Bussink J, Savelkouls $K$, et al. The unfolded protein response protects human tumor cells during hypoxia through regulation of the autophagy genes MAP1LC3B and ATG5. J Clin Invest. 2010;120(1):127-41.

39. Sato K, Tsuchihara K, Fujii S, Sugiyama M, Goya T, Atomi Y, et al. Autophagy is activated in colorectal cancer cells and contributes to the tolerance to nutrient deprivation. Cancer Res. 2007;67(20):9677-84

40. Moreau K, Luo S, Rubinsztein DC. Cytoprotective roles for autophagy. Curr Opin Cell Biol. 2010;22(2):206-11.

41. Jin S, White E. Role of autophagy in cancer: management of metabolic stress. Autophagy. 2007;3(1):28-31.

42. Eng CH, Abraham RT. Glutaminolysis yields a metabolic by-product that stimulates autophagy. Autophagy. 2010;6(7):968-70.

43. Liao XH, Majithia A, Huang X, Kimmel AR. Growth control via TOR kinase signaling, an intracellular sensor of amino acid and energy availability, with crosstalk potential to proline metabolism. Amino Acids. 2008;35(4):761-70.

44. Kumar SH, Rangarajan A. Simian virus 40 small T antigen activates AMPK and triggers autophagy to protect cancer cells from nutrient deprivation. J Virol. 2009;83(17):8565-74.
45. Marambio P, Toro B, Sanhueza C, Troncoso R, Parra V, Verdejo H, et al. Glucose deprivation causes oxidative stress and stimulates aggresome formation and autophagy in cultured cardiac myocytes. Biochim Biophys Acta. 2010;1802(6):509-18.

46. Kang R, Tang D, Schapiro NE, Livesey KM, Farkas A, Loughran P, et al. The receptor for advanced glycation end products (RAGE) sustains autophagy and limits apoptosis, promoting pancreatic tumor cell survival. Cell Death Differ. 2010:17(4):666-76.

47. Ruderman NB, Xu XJ, Nelson L, Cacicedo JM, Saha AK, Lan F, et al. AMPK and SIRT1: a long-standing partnership? Am J Physiol Endocrinol Metab. 2010;298(4):E751-760.

48. Spaeth E, Klopp A, Dembinski J, Andreeff M, Marini F. Inflammation and tumor microenvironments: defining the migratory itinerary of mesenchymal stem cells. Gene Ther. 2008;15(10):730-8.

49. Balkwill F, Mantovani A. Inflammation and cancer: back to Virchow? Lancet. 2001;357(9255):539-45

50. Hussain SP, Harris CC. Inflammation and cancer: an ancient link with novel potentials. Int J Cancer. 2007;121(11):2373-80.

51. Whiteside TL. The tumor microenvironment and its role in promoting tumor growth. Oncogene. 2008;27(45):5904-12.

52. Zamarron BF, Chen W. Dual roles of immune cells and their factors in cancer development and progression. Int J Biol Sci. 2011;7(5):651-8.

53. Hanahan D, Weinberg RA. Hallmarks of cancer: the next generation. Cell. 2011;144(5):646-74.

54. Joven J, Guirro M, Marine-Casado R, Rodriguez-Gallego E, Menendez JA. Autophagy is an inflammation-related defensive mechanism against disease. Adv Exp Med Biol. 2014;824:43-59.

55. Martinez-Outschoorn UE, Trimmer C, Lin Z, Whitaker-Menezes D, Chiavarina B, Zhou J, et al. Autophagy in cancer associated fibroblasts promotes tumor cell survival: Role of hypoxia, HIF1 induction and NFkappaB activation in the tumor stromal microenvironment. Cell Cycle. 2010;9(17):3515-33.

56. Martinez-Outschoorn UE, Balliet RM, Rivadeneira DB, Chiavarina B, Pavlides S, Wang $C$, et al. Oxidative stress in cancer associated fibroblasts drives tumorstroma co-evolution: A new paradigm for understanding tumor metabolism, the field effect and genomic instability in cancer cells. Cell Cycle. 2010;9(16):3256-76.

57. Chiavarina B, Whitaker-Menezes D, Migneco G, Martinez-Outschoorn UE, Pavlides S, Howell A, et al. HIF1-alpha functions as a tumor promoter in cancer associated fibroblasts, and as a tumor suppressor in breast cancer cells: Autophagy drives compartment-specific oncogenesis. Cell Cycle. 2010:9(17):3534-51.

58. Martinez-Outschoorn UE, Whitaker-Menezes D, Lin Z, Flomenberg N, Howell A, Pestell RG, et al. Cytokine production and inflammation drive autophagy in the tumor microenvironment: role of stromal caveolin-1 as a key regulator. Cell Cycle. 2011;10(11):1784-93.

59. Miranti CK, Brugge JS. Sensing the environment: a historical perspective on integrin signal transduction. Nat Cell Biol. 2002;4(4):E83-90.

60. Gilmore AP. Anoikis. Cell Death Differ. 2005;12 Suppl 2:1473-7.

61. Debnath J. Detachment-induced autophagy during anoikis and lumen formation in epithelial acini. Autophagy. 2008;4(3):351-3.

62. Lock R, Debnath J. Extracellular matrix regulation of autophagy. Curr Opin Cell Biol. 2008;20(5):583-8.

63. Tuloup-Minguez V, Greffard A, Codogno P, Botti J. Regulation of autophagy by extracellular matrix glycoproteins in HeLa cells. Autophagy. 2011;7(1):27-39

64. Debnath J, Mills KR, Collins NL, Reginato MJ, Muthuswamy SK, Brugge JS. The role of apoptosis in creating and maintaining luminal space within normal and oncogene-expressing mammary acini. Cell. 2002;111(1):29-40.

65. Fung C, Lock R, Gao S, Salas E, Debnath J. Induction of autophagy during extracellular matrix detachment promotes cell survival. Mol Biol Cell. 2008;19(3):797-806.

66. Chen N, Debnath J. IkappaB kinase complex (IKK) triggers detachmentinduced autophagy in mammary epithelial cells independently of the PI3K-AKT-MTORC1 pathway. Autophagy. 2013;9(8):1214-27.

67. Du J, Teng RJ, Guan T, Eis A, Kaul S, Konduri GG, et al. Role of autophagy in angiogenesis in aortic endothelial cells. Am J Physiol Cell Physiol. 2012;302(2):(383-391.

68. Sachdev U, Cui X, Hong G, Namkoong S, Karlsson JM, Baty CJ, et al. High mobility group box 1 promotes endothelial cell angiogenic behavior in vitro and improves muscle perfusion in vivo in response to ischemic injury. J Vasc Surg. 2012;55(1):180-91. 
69. Kang R, Livesey KM, Zeh HJ, Loze MT, Tang D. HMGB1: a novel Beclin 1-binding protein active in autophagy. Autophagy. 2010;6(8):1209-11.

70. Martinez-Outschoorn UE, Lin Z, Trimmer C, Flomenberg N, Wang C, Pavlides S, et al. Cancer cells metabolically "fertilize" the tumor microenvironment with hydrogen peroxide, driving the Warburg effect: implications for PET imaging of human tumors. Cell Cycle. 2011;10(15):2504-20.

71. Capparelli C, Guido C, Whitaker-Menezes D, Bonuccelli G, Balliet R, Pestell $\mathrm{TG}$, et al. Autophagy and senescence in cancer-associated fibroblasts metabolically supports tumor growth and metastasis via glycolysis and ketone production. Cell Cycle. 2012;11(12):2285-302.

72. Marino ML, Pellegrini P, Di Lernia G, Djavaheri-Mergny M, Brnjic S, Zhang X, et al. Autophagy is a protective mechanism for human melanoma cells under acidic stress. J Biol Chem. 2012;287(36):30664-76.

73. Folkman J. Angiogenesis and apoptosis. Semin Cancer Biol. 2003;13(2):159-67.

74. Lum JJ, Bauer DE, Kong M, Harris MH, Li C, Lindsten T, et al. Growth factor regulation of autophagy and cell survival in the absence of apoptosis. Cell. 2005;120(2):237-48.

75. Qu X, Yu J, Bhagat G, Furuya N, Hibshoosh H, Troxel A, et al. Promotion of tumorigenesis by heterozygous disruption of the beclin 1 autophagy gene. J Clin Invest. 2003;112(12):1809-20.

76. Yue Z, Jin S, Yang C, Levine AJ, Heintz N. Beclin 1, an autophagy gene essential for early embryonic development, is a haploinsufficient tumor suppressor. Proc Natl Acad Sci U S A. 2003;100(25):15077-82.

77. Liang XH, Jackson S, Seaman M, Brown K, Kempkes B, Hibshoosh H, et al. Induction of autophagy and inhibition of tumorigenesis by beclin 1 . Nature. 1999;402(6762):672-6

78. Thorburn J, Frankel AE, Thorburn A. Regulation of HMGB1 release by autophagy. Autophagy. 2009;5(2):247-9.

79. White E, DiPaola RS. The double-edged sword of autophagy modulation in cancer. Clin Cancer Res. 2009;15(17):5308-16.

80. Degterev A, Yuan J. Expansion and evolution of cell death programmes. Nat Rev Mol Cell Biol. 2008;9(5):378-90.

81. Shen HM, Codogno P. Autophagy is a survival force via suppression of necrotic cell death. Exp Cell Res. 2012;318(11):1304-8.

82. Munoz-Gamez JA, Rodriguez-Vargas JM, Quiles-Perez R, Aguilar-Quesada R, Martin-Oliva D, de Murcia G, et al. PARP-1 is involved in autophagy induced by DNA damage. Autophagy. 2009;5(1):61-74.

83. Pikarsky E, Porat RM, Stein I, Abramovitch R, Amit S, Kasem S, et al. NFkappaB functions as a tumour promoter in inflammation-associated cancer. Nature. 2004:431(7007):461-6.

84. Massey DC, Parkes M. Genome-wide association scanning highlights two autophagy genes, ATG16L1 and IRGM, as being significantly associated with Crohn's disease. Autophagy. 2007;3(6):649-51.

85. Saitoh T, Fujita N, Jang MH, Uematsu S, Yang BG, Satoh T, et al. Loss of the autophagy protein Atg16L1 enhances endotoxin-induced IL-1 beta production. Nature. 2008:456(7219):264-8.

86. Zhou R, Yazdi AS, Menu P, Tschopp J. A role for mitochondria in NLRP3 inflammasome activation. Nature. 2011;469(7329):221-5.

87. Bulua AC, Simon A, Maddipati R, Pelletier M, Park H, Kim KY, et al. Mitochondrial reactive oxygen species promote production of proinflammatory cytokines and are elevated in TNFR1-associated periodic syndrome (TRAPS). J Exp Med. 2011;208(3):519-33.

88. Walmsley SR, Print C, Farahi N, Peyssonnaux C, Johnson RS, Cramer T, et al. Hypoxia-induced neutrophil survival is mediated by HIF-1alpha-dependent NF-kappaB activity. J Exp Med. 2005;201(1):105-15.

89. Mihalache CC, Yousefi S, Conus S, Villiger PM, Schneider EM, Simon HU. Inflammation-associated autophagy-related programmed necrotic death of human neutrophils characterized by organelle fusion events. J Immunol. 2011;186(11):6532-42.

90. Naldini A, Morena E, Pucci A, Miglietta D, Riboldi E, Sozzani S, et al. Hypoxia affects dendritic cell survival: role of the hypoxia-inducible factor-1alpha and lipopolysaccharide. J Cell Physiol. 2012;227(2):587-95.

91. Levine B, Deretic V. Unveiling the roles of autophagy in innate and adaptive immunity. Nat Rev Immunol. 2007;7(10):767-77.

92. Schmid D, Munz C. Innate and adaptive immunity through autophagy. Immunity. 2007;27(1):11-21.

93. Pua HH, Dzhagalov I, Chuck M, Mizushima N, He YW. A critical role for the autophagy gene Atg5 in T cell survival and proliferation. J Exp Med. 2007;204(1):25-31.
94. Hubbard VM, Valdor R, Patel B, Singh R, Cuervo AM, Macian F. Macroautophagy regulates energy metabolism during effector $T$ cell activation. J Immunol. 2010;185(12):7349-57.

95. Jia W, He YW. Temporal regulation of intracellular organelle homeostasis in T lymphocytes by autophagy. J Immunol. 2011;186(9):5313-22.

96. Viry E, Paggetti J, Baginska J, Mgrditchian T, Berchem G, Moussay E, et al. Autophagy: an adaptive metabolic response to stress shaping the antitumor immunity. Biochem Pharmacol. 2014;92(1):31-42.

97. Kimmelman AC. The dynamic nature of autophagy in cancer. Genes Dev. 2011;25(19):1999-2010.

98. Wang RC, Wei Y, An Z, Zou Z, Xiao G, Bhagat G, et al. Akt-mediated regulation of autophagy and tumorigenesis through Beclin 1 phosphorylation. Science. 2012;338(6109):956-9.

99. Marino G, Salvador-Montoliu N, Fueyo A, Knecht E, Mizushima N, Lopez-Otin C. Tissue-specific autophagy alterations and increased tumorigenesis in mice deficient in Atg4C/autophagin-3. J Biol Chem. 2007;282(25):18573-83.

100. Liang C, Feng P, Ku B, Dotan I, Canaani D, Oh BH, et al. Autophagic and tumour suppressor activity of a novel Beclin1-binding protein UVRAG. Nat Cell Biol. 2006;8(7):688-99.

101. Takahashi Y, Coppola D, Matsushita N, Cualing HD, Sun M, Sato Y, et al. Bif-1 interacts with Beclin 1 through UVRAG and regulates autophagy and tumorigenesis. Nat Cell Biol. 2007;9(10):1142-51.

102. Takamura A, Komatsu M, Hara T, Sakamoto A, Kishi C, Waguri S, et al. Autophagy-deficient mice develop multiple liver tumors. Genes Dev. 2011;25(8):795-800.

103. Cadwell K, Liu JY, Brown SL, Miyoshi H, Loh J, Lennerz JK, et al. A key role for autophagy and the autophagy gene Atg1611 in mouse and human intestinal Paneth cells. Nature. 2008;456(7219):259-63.

104. Cadwell K, Patel KK, Komatsu M, Virgin HW, Stappenbeck TS. A common role for Atg16L1, Atg5 and Atg7 in small intestinal Paneth cells and Crohn disease. Autophagy. 2009;5(2):250-2.

105. Sun $K$, Guo XL, Zhao QD, Jing $Y Y$, Kou XR, Xie XQ, et al. Paradoxical role of autophagy in the dysplastic and tumor-forming stages of hepatocarcinoma development in rats. Cell Death Dis. 2013;4:e501.

106. Altman BJ, Jacobs SR, Mason EF, Michalek RD, Maclntyre AN, Coloff JL, et al. Autophagy is essential to suppress cell stress and to allow BCR-Ablmediated leukemogenesis. Oncogene. 2011;30(16):1855-67.

107. Guo JY, Chen HY, Mathew R, Fan J, Strohecker AM, Karsli-Uzunbas G, et al. Activated Ras requires autophagy to maintain oxidative metabolism and tumorigenesis. Genes Dev. 2011;25(5):460-70.

108. Wei H, Wei S, Gan B, Peng X, Zou W, Guan JL. Suppression of autophagy by FIP200 deletion inhibits mammary tumorigenesis. Genes Dev. 2011:25(14):1510-27.

109. Li J, Hou N, Faried A, Tsutsumi S, Kuwano H. Inhibition of autophagy augments 5-fluorouracil chemotherapy in human colon cancer in vitro and in vivo model. Eur J Cancer. 2010;46(10):1900-9.

110. Liu D, Yang Y, Liu Q, Wang J. Inhibition of autophagy by 3-MA potentiates cisplatin-induced apoptosis in esophageal squamous cell carcinoma cells. Med Oncol. 2011;28(1):105-11.

111. Kenific CM, Thorburn A, Debnath J. Autophagy and metastasis: another double-edged sword. Curr Opin Cell Biol. 2010;22(2):241-5.

112. Karantza-Wadsworth V, Patel S, Kravchuk O, Chen G, Mathew R, Jin S, et al. Autophagy mitigates metabolic stress and genome damage in mammary tumorigenesis. Genes Dev. 2007;21(13):1621-35.

113. Mortensen M, Soilleux EJ, Djordjevic G, Tripp R, Lutteropp M, Sadighi-Akha $E$, et al. The autophagy protein Atg7 is essential for hematopoietic stem cell maintenance. J Exp Med. 2011;208(3):455-67.

114. Wang C, Liang CC, Bian ZC, Zhu Y, Guan JL. FIP200 is required for maintenance and differentiation of postnatal neural stem cells. Nat Neurosci. 2013;16(5):532-42.

115. Scaffidi P, Misteli T, Bianchi ME. Release of chromatin protein HMGB1 by necrotic cells triggers inflammation. Nature. 2002;418(6894):191-5.

116. Rabinowitz JD, White E. Autophagy and metabolism. Science. 2010;330 (6009):1344-8

117. Yang S, Kimmelman AC. A critical role for autophagy in pancreatic cancer. Autophagy. 2011;7(8):912-3.

118. Yang $X$, Hou J, Han Z, Wang Y, Hao C, Wei L, et al. One cell, multiple roles: contribution of mesenchymal stem cells to tumor development in tumor microenvironment. Cell \& bioscience. 2013;3(1):5

119. Konopleva M, Konoplev S, Hu W, Zaritskey AY, Afanasiev BV, Andreeff M. Stromal cells prevent apoptosis of AML cells by up-regulation of anti-apoptotic proteins. Leukemia. 2002;16(9):1713-24. 
120. Lwin T, Hazlehurst LA, Li Z, Dessureault S, Sotomayor E, Moscinski LC, et al. Bone marrow stromal cells prevent apoptosis of lymphoma cells by upregulation of anti-apoptotic proteins associated with activation of NF-kappaB (RelB/p52) in non-Hodgkin's lymphoma cells. Leukemia. 2007;21(7):1521-31.

121. Nefedova Y, Landowski TH, Dalton WS. Bone marrow stromal-derived soluble factors and direct cell contact contribute to de novo drug resistance of myeloma cells by distinct mechanisms. Leukemia. 2003;17(6):1175-82.

122. Ame-Thomas P, Maby-El Hajjami H, Monvoisin C, Jean R, Monnier D, CauletMaugendre $S$, et al. Human mesenchymal stem cells isolated from bone marrow and lymphoid organs support tumor B-cell growth: role of stromal cells in follicular lymphoma pathogenesis. Blood. 2007;109(2):693-702.

123. Sanchez CG, Penfornis P, Oskowitz AZ, Boonjindasup AG, Cai DZ, Dhule SS, et al. Activation of autophagy in mesenchymal stem cells provides tumor stromal support. Carcinogenesis. 2011;32(7):964-72.

124. Yang ZJ, Chee CE, Huang S, Sinicrope FA. The role of autophagy in cancer: therapeutic implications. Mol Cancer Ther. 2011:10(9):1533-41.

125. Ruiz-Irastorza G, Ramos-Casals M, Brito-Zeron P, Khamashta MA. Clinical efficacy and side effects of antimalarials in systemic lupus erythematosus: a systematic review. Ann Rheum Dis. 2010;69(1):20-8.

\section{Submit your next manuscript to BioMed Central and take full advantage of:}

- Convenient online submission

- Thorough peer review

- No space constraints or color figure charges

- Immediate publication on acceptance

- Inclusion in PubMed, CAS, Scopus and Google Scholar

- Research which is freely available for redistribution 